\title{
Außen- und Innenreferenzen der Schule
}

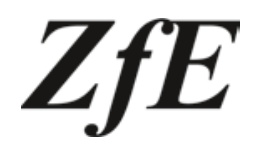

\section{Harm Kuper}

Die erziehungswissenschaftliche Beschreibung der Schule als Organisation erfolgt - cum grano salis - unter zwei getrennten, aber nicht unvereinbaren Prämissen. Sie lassen sich daran unterscheiden, ob der grammatische Singular „Schule“ die einzelne Einrichtung oder den Typus einer Einrichtung bezeichnet. Ist Ersteres der Fall, so beinhaltet der Begriff Schule die Verantwortung der konkreten, einzelnen Schulen in der Formulierung und organisatorischen sowie operativen Ausgestaltung ihrer Programme. Im zweiten Fall liegt im Fokus der Betrachtungen ein Systemgefüge, in dem die einzelnen Schulen Teil einer hierarchisch gegliederten Mehrebenenstruktur und ihre Programme Realisierungen des Systemcodes sind.

In den beiden Prämissen sind neben den begrifflichen Mitteln der wissenschaftlichen Analyse auch normative Vorstellungen zur Lokalisierung der Verantwortung für die Schule verankert. Nicht zuletzt dieser Umstand trägt zur Stabilisierung einer dualistischen Betrachtung der Schule zwischen einzelschulischer Autonomie und gesamtsystemischer Einbettung bei.

Der Themenschwerpunkt dieses Heftes legt eine alternative Betrachtung nahe; sie bezieht sich auf Relationen zwischen der Schule und ihrer Umwelt sowie auf schulinterne Relationen. Damit verschiebt sich die Aufmerksamkeit von der Gegenüberstellung Einzelschule - Schulsystem auf die theoretisch sehr instruktive Überlegung, derzufolge Umwelten als systemrelevante Sachverhalte gelten. Die Autonomie der Selbstregulierung wird folglich zu einer Frage der Spezifikation von Umweltbeziehungen. Die in der Umwelt vorhandenen Bedingungen finden im System Beurteilung und werden zu handlungsrelevanten Ereignissen. Dabei entstehen Abhängigkeiten, sofern aus der Umwelt Ressourcen bezogen werden, und Unsicherheiten, sobald der Umwelt systemrelevante Information entnommen wird. Aus ihnen selektieren Systeme die Strukturen, in denen sie selbst Leistungen erbringen, die sie an ihre Umwelt adressieren. Angewendet auf die Schule geben diese abstrakten Betrachtungen Anlass zu der Frage, welche Systemumwelten für die Schule Bedingungen, aber damit auch Spielräume der internen organisatorischen Gestaltung ihrer pädagogischen Aufgabe geben und welche internen Strukturen

Online publiziert: 23.11 .2012

(C) Springer Fachmedien Wiesbaden 2012

Prof. Dr. H. Kuper $(\bowtie)$

Arbeitsbereich Weiterbildung und Bildungsmanagement, Freie Universität Berlin, Arnimallee 12, 14195 Berlin, Deutschland

E-Mail: harm.kuper@fu-berlin.de 
der Schule zur Verfügung stehen, um ihre pädagogischen Leistungen zu spezifizieren und sicherzustellen.

Die Beiträge zum Themenschwerpunkt greifen Facetten dieser relationalen Betrachtung der Schule auf. Auf der Seite der schulrelevanten Ressourcen bzw. Informationen stehen Berichte aus Studien über die universitäre Lehrerbildung, über vergleichende Schulleistungsuntersuchungen mit Rückmeldefunktion an die Schulen und über die Elternvertretung. Die universitäre Ausbildung von Lehrerinnen und Lehrern ist eine der maßgeblichen Voraussetzungen für die Professionalisierung. Auf ihr beruht die Sicherung professioneller Kompetenz als eine zentrale Ressource der Schule; zudem trägt sie über die Standardisierung von Anforderungen der Ausbildung zur Vergleichbarkeit der organisatorischen Leistungsfähigkeit einzelner Schulen bei. Olga Kunina-Habenicht und Kolleg/inn/en zeigen in einer Delphi-Studie auf, welche bildungswissenschaftlichen Themen der universitären Lehrerbildung von Expertinnen und Experten für bedeutsam erachtet werden. Einen sehr wichtigen Informations-Input für Schulen stellen Vergleichsarbeiten dar. Sie sind ein Instrument der Schulqualitätsentwicklung, insofern sie die an einer Schule geförderten Fachleistungen an externen Maßstäben und empirischen Benchmarks messen. Die Studie von Albrecht Wacker und Jochen Kramer gibt interessante Aufschlüsse darüber, welche Resonanz dieses Instrument in den Schulen des Landes Baden-Württemberg für die Unterrichtsentwicklung findet. Mit der Beteiligung von Eltern an den schulischen Belangen greifen Schulen auf Ressourcen - etwa im Sinne der Mitarbeit, aber auch der Einbindung außerschulischer Lernvoraussetzungen ihrer Schüler - und Informationen gleichermaßen zu. In einer qualitativen Studie legen Stephan Kröner und Mitarbeiter/innen dar, auf welche Voraussetzungen für die Beteiligung an der Elternvertretung sich Schulen bei Eltern mit Migrationshintergrund einstellen können. Sehr klar treten dabei schulinterne Aufgaben der Elternarbeit sowie Gestaltungsoptionen zutage. Mit dem Beitrag von Felicitas Thiel und Kolleginnen erfolgt ein Wechsel von den Input-Relationen zwischen Schule und Umwelt zur Innenreferenz der Schule. Die Autorinnen wenden sich dem Unterricht zu und fragen danach, wie in diesem operativen Kern der Schule Transformationsleistungen prozessual gesteuert werden. Dabei wird deutlich, wie das Klassenmanagement und die Gestaltung von Übergangssituationen im Unterricht auf der professionellen Kompetenz der Lehrkräfte aufbauen. Bettina Kohlrausch und Heike Solga schließlich reichern den Thementeil mit einer Untersuchung an, die am Ausgang der Schule die output-Relation zur Umwelt beispielhaft aufzeigt. Sie legen empirische Befunde zum Übergang von niedersächsischen Hauptschulen in die berufliche Ausbildung vor, aus denen hervorgeht, wie die Beurteilung fachlicher Leistungen und des Arbeitsverhaltens die Chancen der Jugendlichen auf einen Ausbildungsplatz strukturieren. Hervorgehoben wird auch, wie Schulen durch Leistungen, die in Kooperation mit späteren Ausbildungsbetrieben erbracht werden, die Übergangschancen ihrer Absolventinnen und Absolventen begünstigen können.

Die Perspektive auf die Außen- und Innenreferenzen der Schule legt ein flexibles theoretisches Band um die Beiträge. Es kann dazu beitragen, ein relationales Verständnis der Schule zu begründen; die Beiträge stehen beispielhaft für die thematische Vielfalt der Ausgestaltung dieser Relationen. 